\title{
Domination between Traffic Matrices
}

\author{
Gianpaolo Oriolo \\ Dipartimento di Ingegneria dell'lmpresa, Università degli Studi di Roma "Tor Vergata" \\ email: oriolo@disp.uniroma2.it http://www.disp.uniroma2.it/users/oriolo/
}

A traffic matrix $D^{1}$ dominates a traffic matrix $D^{2}$ if any capacity reservation supporting $D^{1}$ supports $D^{2}$ as well. We prove that $D^{1}$ dominates $D^{2}$ if and only if $D^{1}$, considered as a capacity reservation, supports $D^{2}$. We show several generalizations of this result.

Key words: network design; multicommodity flows; robust otpimization

MSC2000 Subject Classification: Primary: 90C27; Secondary: 90B18

OR/MS subject classification: Primary: Networks/Graphs; Secondary: Multicommodity

1. Introduction. A common class of network design problems asks for the installation of capacity (bandwidth) in a directed or undirected network $G(V, E)$ as to support a given set of pairwise traffic demands - a traffic matrix - with some additional constraints (integrality, unsplittable flows, resilience etc.).

A crucial assumption in this model is that of knowing the traffic demands in advance. Unfortunately, measuring and predicting traffic demands are difficult problems. Moreover, in several applications, communication patterns change over time, and therefore we are not given a single static traffic matrix, but instead a set $\mathcal{D}$ of non-simultaneous traffic matrices. Several papers have therefore recently addressed the issues of robustness in network optimization (e.g. Bertsimas and Sim [4) and, in particular, several authors considered robust network design models (see Chekuri et al. [5], Duffield et al. [6], Fingerhut et al. 9], Gupta et al. [10, Italiano et al. [12, Oswald et al. [15]), that is, network design models allowing some degree of flexibility in formulating traffic patterns. Mainly, they deal with the case where $\mathcal{D}$ is implicitly given, often by a set of constraints defining a traffic polytope.

We emphasize that, when dealing with more traffic matrices, the routing might be dynamic, that is, it varies as the traffic matrix changes, or static, that is, we keep the same routing template even if the traffic matrix changes. Static routing, which has also has been referred to as oblivious (see Applegate and Cohen [1] and Azar et al. 2]) or stable (see Ben Ameur and Kerivin [3]), might be convenient since in many network applications "migrating" from a routing to another one is costly (see Ben Ameur and Kerivin [3]). Interestingly, going for a static routing makes sometimes network design problems easier (see Chekuri et al. [5]).

In this paper, we are interested in the case where $\mathcal{D}$ is explicitly given and, in particular, in the "simple" case where $\mathcal{D}=\left\{D^{1}, D^{2}\right\}$. For $h=1,2, d^{h}(i j)$ specifies, for each ordered pair of nodes $i, j \in V$, the value of the expected demand from $i$ to $j$. We consider the following question: when is it possible to discard $D^{2}$ so that $\mathcal{D}$ reduces to $D^{1}$ ? The answer to this question is related to a property of domination between $D^{1}$ and $D^{2}$. We say that a traffic matrix $D^{1}$ dominates a traffic matrix $D^{2}$ if any capacity reservation $U: E \rightarrow \mathbb{R}_{+}$supporting $D^{1}$ supports $D^{2}$ as well. As we show later, this property may be easily expressed in terms of containment between two suitable polyhedra.

Our main result is a good characterization for domination: $D^{1}$ dominates $D^{2}$ if and only if $D^{1}$, considered as a capacity reservation, supports $D^{2}$ (Theorem 2.1). To the best of our knowledge this surprisingly simple result has not been observed before. The theorem extends to the cases where demands are to be routed by either unsplittable or integral flows.

We also consider the case where the routing has to be static. This requires some stronger definitions of domination. In a first one, that we call strong domination, if a capacity reservation $U$ supports $D^{1}$, then there must exist a common routing template for serving both $D^{1}$ and $D^{2}$. In a second one, that we call total domination, if a capacity reservation $U$ supports $D^{1}$, then every routing template serving $D^{1}$ must serve $D^{2}$ as well. Again, these properties may be easily expressed in terms of containment between suitable polyhedra. Both properties have simple good characterizations; in particular $D^{1}$ totally dominates $D^{2}$ if and only if $d^{1}(i j) \geq d^{2}(i j)$ for any demand $d(i j)$ (Theorem 2.5.

We close by discussing the extension of domination to the case where $|\mathcal{D}|=3$. 
1.1 Preliminaries. Even if our results directly extend to the directed case, for sake of simplicity, throughout the paper we suppose that we are given an undirected network $G(V, E)$. $G$ is simple and, without loss of generality, complete, i.e. $E=\{i j: i, j \in V, i \neq j\}$. We let $|V|=n$.

A traffic matrix $D$ is a $n \times n$ symmetric, non-negative, real matrix, where $d(i j)$ is the amount of demand that has to be routed between node $i$ and $j$; we assume $d(i i)=0$ for any $i$. Given its symmetry, we will often regard $D$ as a vector in $\mathbb{R}_{+}^{K}$, where $K=\{i j: i, j \in V\}$ is the set of demands.

A capacity reservation $U \in \mathbb{R}_{+}^{E}$ specifies the amount of capacity $u(e)$ we install over each $\operatorname{arc} e \in E$. It will be often convenient to extend $U$ on $K$ by setting $u(i i)=0$ for any $i$.

A routing $F \in \mathbb{R}_{+}^{K \times E}$ specifies, for each demand $i j \in K$ and arc $e \in E$, the fraction $f_{i j}(e)$ of a unit flow between $i$ and $j$ that is routed through $e$ : if $d(i j)$ demand needs to be routed, then it is routed by simply scaling up the unit flow by $d(i j)$.

A capacity reservation $U$ and a routing $F$ support a traffic matrix $D$ if $D$ may be routed over $G$ equipped with capacity $U$ via $F$, that is, for each $e \in E, \sum_{i j \in K} f_{i j}(e) d(i j) \leq u(e)$. We simply say that $U$ supports $D$ if there exists a routing $F$ such that $U$ and $F$ support $D$. A nice characterization of this latter property is a well-known result from the literature (Iri [11, Onaga and Kakusho [14]).

The metric polytope, a normalization of the metric cone, is defined as follows:

$$
\begin{array}{r}
P_{\mu}=\left\{\mu \in \mathcal{R}_{+}^{K}: \quad \mu(i j)+\mu(j k) \geq \mu(i k) \forall i, j, k \in V ;\right. \\
\left.\sum_{i j \in K} \mu(i j)=1 ; \quad \mu(i i)=0 \forall i \in V\right\}
\end{array}
$$

Theorem 1.1 Iri 11], Onaga and Kakusho [14] A capacity matrix $U$ supports a traffic matrix $D$ if and only $\sum_{i j \in K} \mu(i j) u(i j) \geq \sum_{i j \in K} \mu(i j) d(i j)$ for any $\mu \in P_{\mu}$.

We denote by $\mathcal{D}(U, F)$ the set of traffic matrices that are supported by a capacity reservation $U$ and a routing $F$. That is, $\mathcal{D}(U, F)=\left\{D \in \mathbb{R}_{+}^{K}: \sum_{i j \in K} f_{i j}(e) d(i j) \leq u(e), e \in E\right\}$. We simply denote by $\mathcal{D}(U)$ the set of traffic matrices that are supported by a capacity reservation $U$. By Theorem 1.1 $\mathcal{D}(U)=\left\{D \in \mathbb{R}_{+}^{K}: \sum_{i j \in K} \mu(i j) d(i j) \leq \sum_{i j \in K} \mu(i j) u(i j), \mu \in P_{\mu}\right\}$. It is easy to see that $\mathcal{D}(U)$ is a down-monotone polytope.

Analogously, $\mathcal{U F}(D)$ is the set of capacity reservations and routings supporting a traffic matrix $D$. That is, $\mathcal{U F}(D)=\left\{(U, F) \in \mathbb{R}_{+}^{E} \times \mathbb{R}_{+}^{K \times E}: F\right.$ is a routing and $\left.\sum_{i j \in K} f_{i j}(e) d(i j) \leq u(e), e \in E\right\}$ (the constraint forcing $F$ to define a routing are standard flow constraints). $\mathcal{U}(D)$ is the set of capacity reservations supporting $D$ and by Theorem 1.1. $\mathcal{U}(D)=\left\{U: \sum_{i j \in K} \mu(i j) d(i j) \leq \sum_{i j \in K} \mu(i j) u(i j)\right.$, $\mu \in P_{\mu}$. Moreover, $\mathcal{U}(D)$ is a projection of $\mathcal{U} \mathcal{F}(D)$ and is an up-monotone polyhedron.

2. Domination. Let $D^{1}$ and $D^{2}$ be two traffic matrices. We say that $D^{1}$ dominates $D^{2}$ if $\mathcal{U}\left(D^{1}\right) \subseteq$ $\mathcal{U}\left(D^{2}\right)$. In other words, $D^{1}$ dominates $D^{2}$ if any capacity reservation supporting $D^{1}$ supports also $D^{2}$. Trivially, $D^{1}$ dominates $D^{2}$ if $d^{1}(i j) \geq d^{2}(i j)$ for any demand $i j \in K$, but this condition is only sufficient.

TheOREm $2.1 D^{1}$ dominates $D^{2}$ if and only if $D^{1}$, regarded as a capacity reservation, supports $D^{2}$.

Proof. Necessity. Trivially, $D^{1}$, regarded as a capacity reservation, supports the traffic matrix $D^{1}$. Hence $D^{1}$ must support $D^{2}$ too. Sufficiency. Suppose $D^{1}$, regarded as a capacity reservation, supports the traffic matrix $D^{2}$. By Theorem 1.1 .

$$
\sum_{i j \in K} \mu(i j) d^{1}(i j) \geq \sum_{i j \in K} \mu(i j) d^{2}(i j) \quad \text { for any } \mu \in P_{\mu}
$$

Let $U$ be any capacity reservation supporting $D^{1}$. Again, by Theorem 1.1 . 


$$
\sum_{i j \in K} \mu(i j) u(i j) \geq \sum_{i j \in K} \mu(i j) d^{1}(i j) \quad \text { for any } \mu \in P_{\mu}
$$

and therefore, combining (1) and (2):

$$
\sum_{i j \in K} \mu(i j) u(i j) \geq \sum_{i j \in K} \mu(i j) d^{2}(i j) \quad \text { for any } \mu \in P_{\mu}
$$

that is, $U$ supports $D^{2}$ too.

The previous theorem implies that recognizing if a traffic matrix $D^{1}$ dominates a traffic matrix $D^{2}$ is easy, since it is equivalent to solve a fractional multi-commodity problem. We also recall that recognizing if a polyhedron contains another polyhedron is easy when both polyhedra are given by systems of inequalities (see Eaves and Freund [7]), but, in our case, the size of the systems describing $\mathcal{U}\left(D^{1}\right)$ and $\mathcal{U}\left(D^{2}\right)$ is not polynomially bounded in the size of the input.

2.1 Unsplittable flows. A routing $F$ is unsplittable if it routes each demand $d(i j)$ on a single path from $i$ to $j$. Mare precisely, a routing $F$ is unsplittable if, for each demand $i j \in K$ and each arc $e \in E$, $f_{i j}(e) \in\{0,1\}$.

We say that $U$ supports $D$ with unsplittable flows if there exists an unsplittable routing $F$ such that $U$ and $F$ support $D$. If $D^{1}$ and $D^{2}$ are two traffic matrices, we say that $D^{1}$ dominates $D^{2}$ with respect to unsplittable flows if any capacity $U$ supporting $D^{1}$ with unsplittable flows also supports $D^{2}$ with unsplittable flows.

THEOREM $2.2 D^{1}$ dominates $D^{2}$ with respect to unsplittable flows if and only if $D^{1}$, regarded as a capacity reservation, supports $D^{2}$ with unsplittable flows.

Proof. Necessity. Trivially, $D^{1}$, regarded as a capacity reservation, supports the traffic matrix $D^{1}$ with unsplittable flows. Hence $D^{1}$ must support $D^{2}$ with unsplittable flows too.

Sufficiency. Suppose $D^{1}$, regarded as a capacity reservation, supports the traffic matrix $D^{2}$ with unsplittable flows. Then there exists an unsplittable routing $F$ such that $\sum_{r s \in K} f_{r s}(i j) d^{2}(i j) \leq d^{1}(i j)$, for each arc $i j \in E$. Also let $U$ be any capacity supporting $D^{1}$ with unsplittable flows; then there exists an unsplittable routing $Q$ such that $\sum_{i j \in K} q_{i j}(g) d^{1}(i j) \leq u(g)$, for each arc $g \in E$.

We must show that there exists an unsplittable routing $W$ such that $U$ and $W$ support $D^{2}$. We define $W$ as follows:

$$
w_{r s}(g)=\sum_{i j \in K} q_{i j}(g) f_{r s}(i j) \quad \text { for each demand } r s \in K \text { and each arc } g
$$

It is easy to see that $W$ defines an unsplittable routing. Moreover, for any $g \in E$ :

$$
\begin{aligned}
& \sum_{r s \in K} d^{2}(r s) w_{r s}(g)=\sum_{r s \in K} d^{2}(r s) \sum_{i j \in K} q_{i j}(g) f_{r s}(i j)= \\
= & \sum_{i j \in K} q_{i j}(g) \sum_{r s \in K} f_{r s}(i j) d^{2}(r s) \leq \sum_{i j \in K} q_{i j}(g) d^{1}(i j) \leq u(g)
\end{aligned}
$$

We point out that, since recognizing if a capacity reservation $U$ supports a traffic matrix $D$ with unsplittable flows (see Kleinberg [13]) is NP-complete, the previous theorem does not give a good characterization. On the contrary, it shows that recognizing when a traffic matrix $D^{1}$ dominates a traffic matrix $D^{2}$ with respect to unsplittable flows is NP-complete. 
2.2 Integral flows. We say that a capacity reservation $U$ supports a (integral) traffic demand $D$ with integral flows if there exists a routing $F$ such that $U$ and $F$ support $D$ and $f_{i j}(e) d(i j)$ is integral for each demand $i j \in K$ and each arc $e \in E$.

If $D^{1}$ and $D^{2}$ are two traffic matrices, we say that $D^{1}$ dominates $D^{2}$ with respect to integral flows if any capacity reservation $U$ supporting $D^{1}$ with integral flows also supports $D^{2}$ with integral flows. The proof of the following theorem is similar to that of Theorem 2.2. so we omit it.

THEOREm $2.3 D^{1}$ dominates $D^{2}$ with respect to integral flows if and only if $D^{1}$, regarded as a capacity reservation, supports $D^{2}$ with integral flows.

Again, since recognizing if a capacity matrix $U$ supports a traffic matrix $D$ with integral flows is NPcomplete then such is the complexity of checking if a traffic matrix $D^{1}$ dominates a traffic matrix $D^{2}$ with respect to integral flows.

2.3 Strong domination. We have emphasized in Section 1 that, when dealing with more traffic matrices, the routing might be either dynamic, i.e. it varies as the traffic matrix changes, or static, i.e. we always keep the same routing. Our first definition of domination does not take into account routing, allowing therefore for a dynamic one. We now discuss stronger properties of domination that allows to deal with static routing.

Let $D^{1}$ and $D^{2}$ be two traffic matrices. We say that $D^{1}$ strongly dominates $D^{2}$ if $\mathcal{U}\left(D^{1}\right) \subseteq\left(\mathcal{U F}\left(D^{1}\right) \cap\right.$ $\left.\mathcal{U F}\left(D^{2}\right)\right)\left.\right|_{U}$, where $\left.\right|_{U}$ denotes the projection over the $U$-space. In other words, for any capacity reservation $U$ supporting $D^{1}$, there exists a same routing $F$ such that $U$ and $F$ support both $D^{1}$ and $D^{2}$. Trivially, if $D^{1}$ strongly dominates $D^{2}$, then $D^{1}$ dominates $D^{2}$.

Suppose now that $D^{1}$ dominates $D^{2}$. Therefore, from Theorem 2.1 there exists a routing $F$ such that $D^{1}$ and $F$ support $D^{2}$. For $h=1,2$, let $I\left(D^{h}\right)=\left\{i j \in K: d^{h}(i j)>0\right\}$. We will prove that, if $F$ is such that each demand $i j \in I\left(D^{1}\right) \cap I\left(D^{2}\right)$ is directly routed on the link $e \equiv i j$, i.e. $f_{i j}(i j)=1$ for any $i j \in I\left(D^{1}\right) \cap I\left(D^{2}\right)$, then $D^{1}$ strongly dominates $D^{2}$. In fact, we will prove that the existence of such a routing is also necessary for $D^{1}$ to strongly dominate $D^{2}$.

It is therefore convenient to define:

$$
\begin{gathered}
\bar{D}^{1}: \quad \bar{d}^{1}(i j)= \begin{cases}d^{1}(i j)-d^{2}(i j) & \text { if } i j \in I\left(D^{1}\right) \cap I\left(D^{2}\right) \\
d^{1}(i j) & \text { else }\end{cases} \\
\bar{D}^{2}: \quad \bar{d}^{2}(i j)= \begin{cases}0 & \text { if } i j \in I\left(D^{1}\right) \cap I\left(D^{2}\right) \\
d^{2}(i j) & \text { else }\end{cases}
\end{gathered}
$$

THEOREM $2.4 D^{1}$ strongly dominates $D^{2}$ if and only if both the following statements hold:

(i) $d^{1}(i j) \geq d^{2}(i j)$ for any $i j \in I\left(D^{1}\right) \cap I\left(D^{2}\right)$;

(ii) $\bar{D}^{1}$, regarded as a capacity reservation, supports $\bar{D}^{2}$.

Proof. Necessity. As usual, $D^{1}$, regarded as a capacity reservation, supports the traffic matrix $D^{1}$. Since $D^{1}$ strongly dominates $D^{2}$, it follows that there must exist a routing $F$ such that $D^{1}$, regarded as a capacity reservation, and $F$ support both $D^{1}$ and $D^{2}$.

Observe that the only routing $\bar{F}$ such that $D^{1}$ and $\bar{F}$ support $D^{1}$ is such that each demand $i j \in I\left(D^{1}\right)$ is directly routed on the link $e \equiv i j$, i.e., for each $i j \in I\left(D^{1}\right)$ :

$$
\bar{F}_{i j}: \quad \bar{f}_{i j}(e)= \begin{cases}1 & \text { if } e \equiv i j \\ 0 & \text { else }\end{cases}
$$

Since $I\left(D^{1}\right) \cap I\left(D^{2}\right) \subseteq I\left(D^{1}\right)$ and $D^{1}$ and $\bar{F}$ must support $D^{2}$, it follows that each demand $i j \in$ $I\left(D^{1}\right) \cap I\left(D^{2}\right)$ is directly routed on the link $e \equiv i j$ and therefore $d^{1}(i j) \geq d^{2}(i j)$. Moreover, the spare capacity $\bar{D}^{1}$ must support $\bar{D}^{2}$. 
Sufficiency. Let $U \in \mathcal{U}\left(D^{1}\right)$ and let $G$ be a routing such that $U$ and $G$ support $D^{1}$. By hypothesis there exists a routing $F$ such that $\bar{D}^{1}$ and $F$ support $\bar{D}^{2}$.

In order, to prove our statement we have to show that there exists a routing $W$ such that $U$ and $W$ support both $D^{1}$ and $D^{2}$. We define $W$ as follows:

$$
W: \quad w_{i j}(e)= \begin{cases}g_{i j}(e) & \text { if } i j \in I\left(D^{1}\right) \text { and } e \in E \\ \sum_{u v \in K} f_{i j}(u v) g_{u v}(e) & \text { if } i j \in I\left(D^{2}\right) \backslash I\left(D^{1}\right) \text { and } e \in E \\ 1 & \text { if } i j \notin I\left(D^{1}\right) \cup I\left(D^{2}\right) \text { and } e \equiv i j \\ 0 & \text { if } i j \notin I\left(D^{1}\right) \cup I\left(D^{2}\right) \text { and } e \neq \equiv i j\end{cases}
$$

It is easy to see that $W$ defines a routing. Since $w_{i j}=g_{i j}$ for any $i j \in I\left(D^{1}\right)$, it follows that $U$ and $W$ support $D^{1}$. Moreover, for any $e \in E$ :

$$
\begin{aligned}
& \sum_{i j \in K} d^{2}(i j) w_{i j}(e)=\sum_{i j \in I\left(D^{1}\right) \cap I\left(D^{2}\right)} d^{2}(i j) w_{i j}(e)+\sum_{i j \in I\left(D^{2}\right) \backslash I\left(D^{1}\right)} \bar{d}^{2}(i j) w_{i j}(e)= \\
& =\sum_{i j \in I\left(D^{1}\right) \cap I\left(D^{2}\right)} d^{2}(i j) g_{i j}(e)+\sum_{i j \in I\left(D^{2}\right) \backslash I\left(D^{1}\right)} \bar{d}^{2}(i j) \sum_{u v \in K} f_{i j}(u v) g_{u v}(e)= \\
& =\sum_{i j \in I\left(D^{1}\right) \cap I\left(D^{2}\right)} d^{2}(i j) g_{i j}(e)+\sum_{u v \in K} g_{u v}(e) \sum_{i j \in I\left(D^{2}\right) \backslash I\left(D^{1}\right)} \bar{d}^{2}(i j) f_{i j}(u v) \leq \\
& \leq \sum_{i j \in I\left(D^{1}\right) \cap I\left(D^{2}\right)} d^{2}(i j) g_{i j}(e)+\sum_{u v \in K} \bar{d}^{1}(u v) g_{u v}(e)= \\
& =\sum_{i j \in I\left(D^{1}\right) \cap I\left(D^{2}\right)}\left(d^{1}(i j)-\bar{d}^{1}(i j)\right) g_{i j}(e)+\sum_{i j \in I\left(D^{1}\right) \cap I\left(D^{2}\right)} \bar{d}^{1}(i j) g_{i j}(e)+\sum_{i j \notin I\left(D^{1}\right) \cap I\left(D^{2}\right)} \bar{d}^{1}(i j) g_{i j}(e)= \\
& =\sum_{i j \in I\left(D^{1}\right) \cap I\left(D^{2}\right)} d^{1}(i j) g_{i j}(e)+\sum_{i j \notin I\left(D^{1}\right) \cap I\left(D^{2}\right)} d^{1}(i j) g_{i j}(e) \leq u(e)
\end{aligned}
$$

and, therefore, $U$ and $W$ support $D^{2}$ too.

Again, recognizing if a traffic matrix $D^{1}$ strongly dominates a traffic matrix $D^{2}$ is easy, since it is equivalent to solve a fractional multi-commodity problem.

Corollary 2.1 If $D^{1}$ dominates $D^{2}$ and $I\left(D^{1}\right) \cap I\left(D^{2}\right)=\emptyset$, then $D^{1}$ strongly dominates $D^{2}$.

2.4 Total domination. We say that $D^{1}$ totally dominates $D^{2}$ if $\mathcal{U} \mathcal{F}\left(D^{1}\right) \subseteq \mathcal{U} \mathcal{F}\left(D^{2}\right)$, i.e. any pair $(U, F)$ supporting $D^{1}$ also supports $D^{2}$. Trivially, if $D^{1}$ totally dominates $D^{2}$, then $D^{1}$ strongly dominates $D^{2}$.

TheOREM $2.5 D^{1}$ totally dominates $D^{2}$ if and only if $d^{1}(i j) \geq d^{2}(i j)$ for any $i j \in K$.

Proof. Necessity. Let $F$ be the routing:

$$
F: \quad f_{i j}(e)= \begin{cases}1 & e \equiv i j \\ 0 & e \neq \equiv i j .\end{cases}
$$

$D^{1}$, regarded as a capacity reservation, and $F$ support the traffic matrix $D^{1}$. Therefore, they must support $D^{2}$ too. Then, for any $h k \in K, h \neq k$ : 


$$
d^{1}(h k) \geq \sum_{i j \in K} d^{2}(i j) f_{i j}(h k)=d^{2}(h k)
$$

Sufficiency. If $U$ and $F$ support $D^{1}$ and $d^{1}(i j) \geq d^{2}(i j)$ for any $i j \in K$, then they support $D^{2}$ too. In fact, for each $e \in E$ :

$$
u(e) \geq \sum_{i j \in K} d^{1}(i j) f_{i j}(e) \geq \sum_{i j \in K} d^{2}(i j) f_{i j}(e)
$$

3. Domination between three traffic matrices. It is natural to extend domination to more traffic matrices, e.g. three. Namely, $D^{1}$ and $D^{2}$ dominate $D^{3}$ if $\mathcal{U}\left(D^{1}\right) \cap \mathcal{U}\left(D^{2}\right) \subseteq \mathcal{U}\left(D^{3}\right)$, that is, any capacity supporting both $D^{1}$ and $D^{2}$ supports $D^{3}$ too. Unfortunately, we have not been able to generalize Theorem 2.1 to this case, and we do not know whether recognizing this kind of domination is easy. We leave this question open, but discuss some necessary or sufficient conditions in the following.

Sufficient Conditions. It follows from Theorem 1.1 that $D^{1}$ and $D^{2}$ dominate $D^{3}$ if:

$$
\max \left(\sum_{i j \in K} \mu(i j) d^{1}(i j), \sum_{i j \in K} \mu(i j) d^{2}(i j)\right) \geq \sum_{i j \in K} \mu(i j) d_{i j}^{3}, \forall \mu \in P_{\mu}
$$

but, as we are going to show, there are cases where this condition is not necessary. The following corollary is proved in Eisenbrand et al. [8]:

Corollary 3.1 Eisenbrand et al. [8] Let $G=(V, E)$ be an undirected graph with edge capacity function $u: E \rightarrow \mathbb{R}_{+}$and $s_{1}, s_{2}, r_{1}, r_{2} \in V$. In the following, all demand values are equal to 1 . If there exists a feasible 2-commodity flow for terminal pairs $\left\{s_{1}, r_{1}\right\},\left\{s_{2}, r_{2}\right\}$ and for terminal pairs $\left\{s_{1}, r_{2}\right\},\left\{s_{2}, r_{1}\right\}$, then there also exists a feasible 2-commodity flow for terminal pairs $\left\{s_{1}, s_{2}\right\},\left\{r_{1}, r_{2}\right\}$.

Now suppose that $V=\left\{s_{1}, s_{2}, r_{1}, r_{2}\right\}$. Let $D^{1}, D^{2}, D^{3}$ be such that the only non-zero demands are: $d^{1}\left(s_{1} r_{1}\right)=d^{1}\left(s_{2} r_{2}\right)=d^{2}\left(s_{1} r_{2}\right)=d^{2}\left(s_{2} r_{1}\right)=d^{3}\left(s_{1} s_{2}\right)=d^{3}\left(r_{1} r_{2}\right)=1$. It follows from Corollary 3.1 that $D^{1}$ and $D^{2}$ dominate $D^{3}$. On the other hand, Inequality (4) is not satisfied when we consider $\mu\left(s_{1} r_{1}\right)=\mu\left(s_{2} r_{2}\right)=\mu\left(s_{1} r_{2}\right)=\mu\left(s_{2} r_{1}\right)=1 / 8$ and $\mu\left(s_{1} s_{2}\right)=\mu\left(r_{1} r_{2}\right)=1 / 4$.

Necessary Conditions. $D^{1}$ and $D^{2}$ dominate $D^{3}$ only if:

$$
\sum_{i j \in K} \mu(i j) \max \left(d^{1}(i j), d^{2}(i j)\right) \geq \sum_{i j \in K} \mu(i j) d_{i j}^{3}, \forall \mu \in P_{\mu}
$$

In fact, consider $D: d(i j)=\max \left(d^{1}(i j), d^{2}(i j)\right)$. $D$ dominates both $D^{1}$ and $D^{2}$ by Theorem 2.5 . Therefore, if $D_{1}$ and $D_{2}$ dominate $D^{3}$, then $D$ dominates $D^{3}$ too. Inequality (5) then follows from Theorem 2.1 .

Unfortunately, the condition is not sufficient: consider. Suppose that $V=\{a, b, c\}$. Let $D^{1}, D^{2}, D^{3}$ be such that the only non-zero demands are: $d^{1}(a b)=2, d^{1}(b c)=2, d^{2}(a c)=4, d^{3}(a b)=2 ; d^{3}(b c)=2$; $d^{3}(a c)=3$. Then Inequality (5) is satisfied but $U: u(a b)=2, u(b c)=2, u(a c)=2$ does support $D^{1}$ and $D^{2}$ but not $D^{3}$.

Acknowledgment. We thank Bruce Shepherd for his many helpful comments on the paper.

\section{References}

[1] D. Applegate and E. Cohen, Making intra-domain routing robust to changing and uncertain traffic demands: Understanding fundamental tradeoffs, Proceedings of SIGCOMM 2003, 313-324.

[2] Y. Azar, E. Cohen, A. Fiat, H. Kaplan, and H. Räcke, Optimal oblivious routing in polynomial time, Proceedings of STOC 2003, Journal of Computer System Sciences, 69(3) (2004), 383-394. 
[3] W. Ben-Ameur and H. Kerivin, Routing of uncertain demands, Optimization and Engineering 3 (2005), 283-313.

[4] D. Bertsimas and M. Sim, Robust discrete optimization and network flows, Mathematical Programming, 98(1-3) (2003), 49-71.

[5] C. Chekuri, G. Oriolo, M.G. Scutellá and F.B. Shepherd, Hardness of robust network design, Proceedings of INOC 2005, 455-461.

[6] N.G. Duffield, P. Goyal, A.G. Greenberg, P.P. Mishra, K.K. Ramakrishnan and J.E. van der Merwe, A flexible model for resource management in virtual private networks, Proceedings of SIGCOMM 1999, 95-108.

[7] B.C. Eaves and R.M. Freund, Optimal scaling of balls and polyhedra, Mathematical Programming, 23 (1982), 138-147.

[8] F. Eisenbrand, F. Grandoni, G. Oriolo and M. Skutella, New approaches for virtual private network design, Proceedings of ICALP 2005, 1151-1162.

[9] J. A. Fingerhut, S. Suri and J.S. Turner, Designing least-cost nonblocking broadband networks, Journal of Algorithms, 24(2) (1997), 287-309.

[10] A. Gupta, J. Kleinberg, A. Kumar, R. Rastogi and B. Yener, Provisioning a virtual private network: a network design problem for multicommodity flow, Proceedings of STOC 2001, 389-398.

[11] M. Iri, On an extension of the max-flow min-cut theorem for multicommodity flows, Journal of the Operations Research Society of Japan, 13 (1970), 129-135.

[12] G. Italiano, S. Leonardi and G. Oriolo, Design of trees in the hose model: the balanced case, Operations Research Letters 34(6), 601-606(2006).

[13] J. Kleinberg, Single-source unsplittable flow, Proceedings of FOCS 1996, 68-77.

[14] K. Onaga and O. Kakusho, On feasibility conditions of multicommodity flows in networks; IEEE Transactions of Circuit Theory, CT-18 (1970), 425-429.

[15] P. Oswald, F.B. Shepherd, P. Winzer and M. Zirngibl, Robust network design and selective randomized load balancing, Proceedings of ECOC 2005, 1-23. 